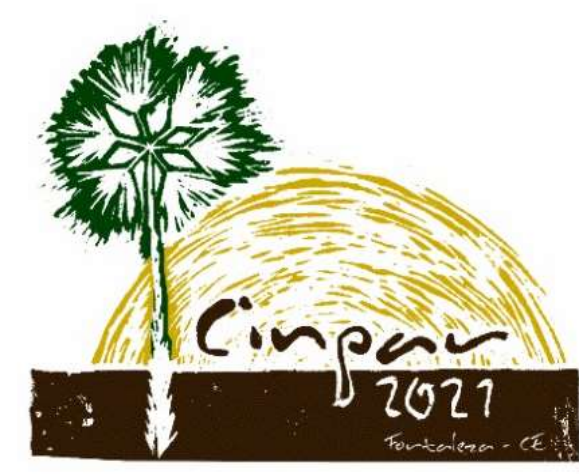

XVII Congresso Internacional sobre Patologia e Reabilitação das Construções

XVII Congreso Internacional sobre Patología y Rehabilitación de las Construcciones

XVII International Conference on Pathology and Constructions Rehabilitation

FORTALEZA (Brasil), 3 a 5 de junho de 2021

https://doi.org/10.4322/CINPAR.2021.055

\title{
EFEITO DE ADITIVOS SUPERPLASTIFICANTES NAS PROPRIEDADES MECÂNICAS DE BARRAS DE ARGAMASSA SUBMETIDAS A ENSAIO DE EXPANSÃO DA RAA
}

\section{Effect of superplasticizers admixtures on the mechanical properties of mortar bars submitted expansion test of alkali-aggregate reaction}

\author{
Cláudia Flaviana Cavalcante da Silva ${ }^{1}$. Arnaldo Manoel Pereira Carneiro ${ }^{2}$ \\ ${ }^{1}$ Universidade Federal de Pernambuco, Brasil, claudia.cavalcante@caruaru.ifpe.edu.br \\ 2 Universidade Federal de Pernambuco, Brasil, ampc@ufpe.br
}

\begin{abstract}
The alkali-aggregate reaction (AAR) requires the joint action of water, reactive aggregate and alkalis, which can originate from several sources. There are gaps in AAR knowledge on important issues, such as negative or positive contribution of superplasticizers to this pathological manifestation. This study investigates the effect of AAR on the machanical properties of mortar bars, using superplasticizers admixtures, after these bars have been submitted expansion test of alkali-aggregate reaction. The tests used was accelerated test of mortar bars of the RAA, scanning electron microscopy, compressive strength, tensile strength in flexion. It was used a pozzolanic admixtures silica fume, Portland cement CPV ARI and the minimum, optimum and maximum content of lignosulfonate and melamine admixtures. It was observed in both types of additives that the higher the content of these additives, the greater the results of the compressive strength and the greater the results of the expansion of the mortar bars. It is observed that the tensile strength in flexion didn't correlate with the expansion and with that it is noticed that the cracks caused in the specimens by the expansion test, don't significantly interfere in the tensile strength in flexion. The type and content of superplasticizer admixtures interferes with the expansion of AAR.
\end{abstract}

Keywords: superplasticizers admixtures; alkali-aggregate reaction; mechanical properties.

\section{Introdução}

O concreto é um material de construção bastante utilizado em todo o mundo e está sujeito a problemas patológicos, originados por seus materiais constituintes e pelo ambiente em que se encontra inserido.

Para desencadear a RAA no concreto é necessária a presença de constituintes reativos dos agregados, elevada alcalinidade e umidade suficiente. Esta reação se origina tipicamente com uma borda de gel ao redor dos agregados, esses géis aumentam de volume ao absorver água, provocando a expansão e fissuras do concreto (CHANG; MICHAEL; JASON, 2018; NAGROCKIENĖ; RUTKAUSKAS, 2019).

A principal fonte dos álcalis no concreto é o cimento Portland, embora seus constituintes tais como adições minerais, alguns agregados e aditivos químicos, possam contribuir na sua alcalinidade.

Existem várias maneiras de mitigação da RAA, incluindo o uso de adições minerais, porém este mecanismo de mitigação ainda não encontra-se totalmente elucidado (HASHEMI et al., 2015; CHANG, MICHAEL, JASON, 2018; GAUTAM, et al., 2017). Atualmente, o metacaulim, a sílica ativa e cinza volante têm mostrado satisfatória atividade pozolânica no combate a RAA. Tem-se conhecimento de que o uso de algumas adições minerais requer elevada relação água/aglomerante, porém o elevado consumo de água afeta algumas propriedades no estado fresco e endurecido, acarretando, dentre outros fatores, a redução da resistência 
mecânica e consequentemente da durabilidade das argamassas e concretos. Como o elevado teor de água prejudica estes fatores no concreto e na argamassa, tem-se conhecimento da necessidade da utilização de aditivos químicos, visando à obtenção da consistência requerida da massa fresca, sem que aumente o teor de água.

A pozolana sílica ativa necessita ser bem dispersa na massa fresca com uso de aditivos químicos, pois quando o sistema não se torna bem disperso, as partículas de sílica ativa tendem a aglomerar-se, podendo provocar a RAA, pois essas partículas aglomeradas fazem a função do agregado reativo. A fim de amenizar a ocorrência desses fatores prejudiciais ao concreto, se recomenda $\mathrm{o}$ uso de aditivos químicos como os superplastificantes.

Sabendo-se que a adsorção do aditivo superplastificante acarreta a dispersão das partículas de materiais cimentícios, esta dispersão interfere na consistência podendo interferir na estabilidade de argamassas e concretos. Essa afirmação induz a pressupor que a consistência e estabilidade no estado fresco, interferem no comportamento da RAA, devido a estas influenciarem a porosidade das argamassas no estado endurecido. De acordo com Ramachandran (1995), o concreto de elevada consistência pode propiciar a perda de ar, reduzindo a quantidade de vazios desse concreto. Segundo Multon (2004), um aumento da porosidade permite que os géis da RAA se formem sem exercer pressão e, consequentemente, sem acarretar fissuraçao.

Os aditivos originam diferentes dispersões das partículas de materiais cimentícios e acarretam alteração das propriedades no estado fresco e endurecido de argamassas e concretos. Como essas diferentes características dos aditivos interferem no comportamento do concreto e argamassa, percebe-se que o seu efeito no comportamento da RAA pode se apresentar diferenciado, podendo interferir de maneira significativa. Como exemplos desse comportamento, encontram-se nos estudos realizados por Farias, Hasparyk e Andrade (2007), Tosun, Felekoglu e Baradan (2007) e Leemann, Lothenbach e Thalmann (2011), em que se observou o aumento ou redução das expansões quando se utilizou os diferente superplastificantes.

Atualmente, ainda existem lacunas no conhecimento da RAA sobre questões importantes, como a contribuição negativa ou positiva dos aditivos superplastificantes para essa menifestação patológica, bem como seu efeito nas propriedades mecânicas quando submetidas aos ensaios de RAA.

Sabe-se que o ensaio de expansão acarreta fissuras nas barras, sendo necessário investigar qual o efeito dessas fissuras de expansão em propriedades mecânicas, como a resistência à tração na flexão e resistência à compressão nessas barras fissuradas. Este estudo tem como objetivo a investigação do efeito da RAA em propriedades mecânicas de barras de argamassa, utilizando aditivos superplastificantes, após essas barras serem submetidas ao ensaio de expanção da reação álcali-agregado.

\section{Materiais e métodos}

\subsection{Materiais}

A adição mineral selecionada neste estudo foi a sílica ativa, com teor de $15 \%$ em substituição ao cimento. A escolha da sílica ativa foi devido ao fato deste material apresentar uma das maiores mitigações, dentre as pozolanas utilizadas por alguns autores, em suas investigações de mitigação da RAA. A sílica ativa é constituída de um pó fino, proveniente da indústria siderúrgica.

O agregado selecionado foi o Milonito, proveniente de uma pedreira localizada na cidade de Vitória de Santo Antão - PE, tendo como principal mineralogia deletéria o quartzo microcristalino. A escolha do agregado foi devido a este apresentar maior reatividade dentre os vários tipos investigados por Silva, Monteiro e Gusmão, (2011). A rocha foi coletada na própria pedreira, onde os maciços rochosos já haviam sido demolidos e encontrava-se amontoada para posterior britagem. A rocha foi triturada visando à obtenção da granulometria requerida para os ensaios.

O cimento utilizado foi o CPV ARI que apresenta baixa alcalinidade, por não possuir material pozolânico, o que possibilita maior identificação da influência dos tipos de adições minerais no processo de expansão, sem interferência de adições presentes no cimento, e pelo motivo do CPV ARI fazer parte da lista de cimentos 
recomendado para realização do ensaio de expansão. Este cimento pode conter até $5 \%$ de material carbonático (fíller calcáreo).

O aditivo químico escolhido foi o superplastificante (comercial) por ser atualmente muito utilizado na construção civil e recomendado por várias normas, inclusive a brasileira, para uso em ensaios laboratoriais em que se investiga a mitigação da RAA com uso de adições minerais. Foi utilizado aditivo lignosulfonato (LI) e melamina (ME) com teores mínimos, ótimos e máximos. Os teores mínimo e máximos foram os recomendados pelo fabricante, e o teor ótimo de cada aditivo foi optido pela realização de ensaios utilizando o cone de Marsh.

\subsection{Métodos de ensaios}

\subsubsection{Métodos de ensaios para caracterização dos materiais}

$O$ ensaio de difração de raio-X foi utilizado para caracterização da sílica ativa. No difratograma, a radiação $X$ usada foi $K \alpha$ do cobalto nas seguintes condições de ensaio: faixa de varredura de $2 \theta\left(15^{\circ}\right.$ a $\left.60^{\circ}\right)$, velocidade de varredura de $0,05^{\circ} 2 \theta / \mathrm{s}$, tensão de filamento de $35 \mathrm{Kv}$ e corrente de filamento de $45 \mathrm{~mA}$. Sabe-se que a presença de halo amorfo acarreta elevada pozolanicidade do material. A análise petrográfica da amostra de rocha foi realizada utilizando microscópio estereoscópio e óptico, em que investigou-se a granulação, características mineralógicas, textura, estrutura e potencialidade reativa desse agregado, através da NBR 15577-3. Foi utilizado o ensaio de fluorescência de raio-X para análise química do cimento, agregado, aditivos químicos e da sílica ativa. Foi realizada com a sílica ativa o ensaio de atividade pozolânica aos 28 dias.

\subsubsection{Ensaio acelerado das barras de argamassa (NBR 15577-5)}

Este ensaio é bastante utilizado para analisar o efeito da possibilidade de mitigação quando se faz uso das adições minerais. Nas argamassas, a relação água/cimento foi 0,47 e o traço 1:2,25 (cimento/agregado), conforme recomenda o método acelerado das barras de argamassa descrito na NBR 15577-5. Utilizou-se neste ensaio o teor de $15 \%$ de sílica ativa em substituição ao cimento. Os teores dos dois tipos de aditivos foram: lignosulfonato (mínimo 0,6\%, ótimo 0,8\%, máximo 1,0\%); Melamina (mínimo 0,5\%, ótimo 1,0\%, máximo 1,5\%). A dosagem dos aditivos foi expressa em porcentagem da massa de material cimentício, conforme recomenda a NBR 11768 (ABNT, 2011), considerando o teor de sólido desses aditivos. A água contida nos aditivos químicos foi considerada no cálculo da relação água/materiais cimentícios, devido à recomendação da NBR 15577-5. Optou-se por utilizar um tipo de cimento, bem como um único agregado considerado potencialmente reativo. Diante do fato da argamassa apresentar, na idade de 30 dias, comportamento de expansão proporcional ao comportamento na idade de 16 dias, e por motivo de alguns ensaios deste estudo necessitarem fazer uso dos corpos de prova do ensaio de expansão que foi estendido aos 30 dias, optou-se por investigar os demais ensaios (resistência à compressão, tração na flexão e microscopia eletrônica de varredura ) na idade de 30 dias. As barras de argamassa apresentam dimensões de $(2,5 \times 2,5 \times 28,5) \mathrm{cm}$, totalizando 21 barras.

\subsubsection{Ensaio de resistência à compressão e resistência à tração}

As barras de argamassa que foram submetidas ao ensaio de expansão na idade de 30 dias (finalizado esse ensaio nessa idade), foram utilizadas em ensaios, incluindo o de resistência à compressão e de tração na flexão. Por motivo deste estudo intencionar a investigação da resistência à tração na flexão e compressão dos corpos de prova que foram submersos em soda no ensaio de expansão da RAA, foi necessário seccionar as barras do ensaio de expansão para obtenção de corpos de prova para estes dois ensaios mecânicos. Para o ensaio de resistência à compressão, essas barras foram seccionadas em formato cúbicos $\left(2,5 \mathrm{~cm}^{3}\right)$, sendo obtido 4 cubos (corpos de prova) de cada tipo de argamassa. E para o ensaio de resistência à tração na flexão, as barras foram seccionadas ao meio, obtendo-se corpo de prova em formato prismático com dimensões $(2,5 \times 2,5 \times 142,5) \mathrm{cm}$, sendo obtidos 4 prismas de cada tipo de argamassa. $O$ cubo e o prisma podem ser visualizados na Figura 1. 


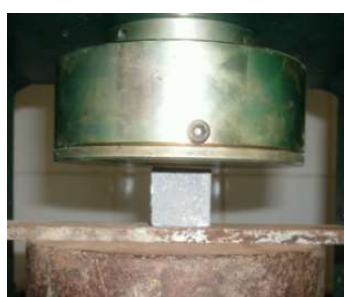

(a)

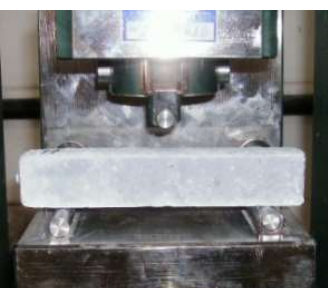

(b)

Figura 1 - Corpo de prova cúbico (a); Corpo de prova prismático (b).

\subsubsection{Microscopia eletrânica de varredura (MEV)}

As amostras de argamassa foram investigadas através do MEV e EDS (Espectroscopia de Energia Dispersiva), utilizando o detector de elétrons secundários. Uma barra de argamassa de cada traço foi fraturada em dois locais, e em seguida a face oposta de cada fratura foi cortada, formando uma amostra com aproximadamente $5 \mathrm{~mm}$ de altura. Antes da realização do ensaio, as amostras foram metalizadas em vácuo de 10-4 (Pa), recebendo uma espessura de aproximadamente $10 \mathrm{~nm}$ de ouro, no intuito de otimizar as imagens. As argamassas com teor máximo de cada aditivo superplastificante foram investigadas na idade de 30 dias.

\section{Resultados e discussões}

A Figura 2 apresenta o difratograma da sílica ativa, em que se observa halo bastante acentuado, caracterizando um material pozolânico com elevada reatividade. A Tabela 1 mostra as características químicas e mecânicas desta adição mineral em que observa-se que a sílica ativa apresentou um elevado índice de atividade pozolânica (88\%), que pode ser justificado pela elevada superfície específica que acarreta maior facilidade de reação com os compostos. Esse resultado da pozolanicidade corrobora com o resultado desse material obtido no difratômetro, em que o halo é bastante acentuado. Essa adição mineral apresentou uma alcalinidade considerável, no valor de 0,21. A alcalinidade solúvel do cimento Portland foi de 0,54, conforme se observa na Tabela 2, em que mostra as propriedades físicas, mecânicas e químicas.

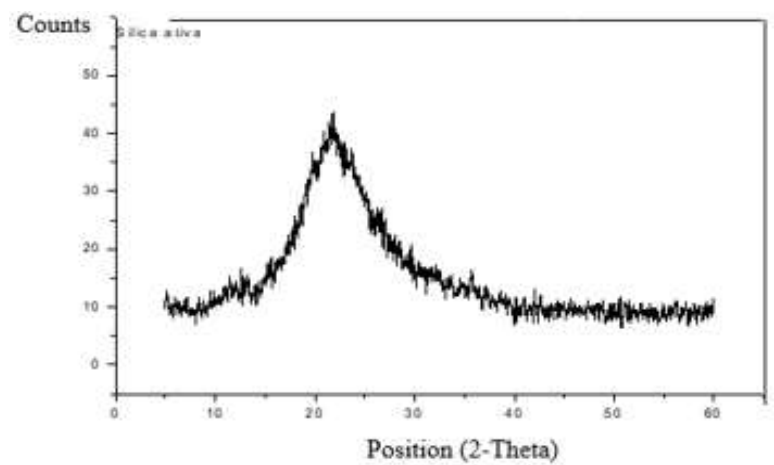

Figura 2 - Difratograma da sílica ativa.

Tabela 1 - Características químicas e mecânicas da adição mineral.

\begin{tabular}{c|c|c|c}
\hline Propriedades químicas (\%) & Sílica ativa & \multicolumn{2}{|c}{ Exigências de normas da ABNT } \\
\hline Álcalis solúveis (disponíveis) & - & - & \\
\hline Óxido de potássio (K2O) & 0,15 & & \\
\hline Óxido de sódio ( $\left.\mathrm{Na}_{2} \mathrm{O}\right)$ & 0,11 & - & - \\
\hline Equivalente alcalino total ( $\mathrm{Na}_{2} \mathrm{O}$ eq) & 0,21 & - & - \\
\hline Propriedades mecânicas & Sílica ativa & \multicolumn{2}{|c}{ Exigências de normas da ABNT } \\
\hline Pozolanicidade com cimento 28 dias (\%) & 88 & $\geq 75 \%$ & NBR 12653 - sílica ativa \\
\hline NOTA: $\mathrm{Na}_{2} \mathrm{Oeq}=\% \mathrm{Na}_{2} \mathrm{O}+0,658 . \% . \mathrm{K}_{2} \mathrm{O}$
\end{tabular}


Tabela 2 - Características físicas, mecânicas e químicas do cimento.

\begin{tabular}{|c|c|c|c|}
\hline Propriedades físicas e mecânicas & Cimento & \multicolumn{2}{|c|}{ Exigências } \\
\hline Área específica blaine $\left(\mathrm{m}^{2} / \mathrm{kg}\right)$ & 461 & $\geq 300 \mathrm{~m}^{2} / \mathrm{kg}$ & NBR 5733 \\
\hline Massa específica $\left(\mathrm{g} / \mathrm{cm}^{3}\right)$ & 3,21 & - & - \\
\hline Expansibilidade em autoclave (\%) & 0,05 & $<0,20 \%$ & NBR 15577-4 \\
\hline Propriedades químicas determinadas & $\%$ & \multicolumn{2}{|c|}{ Exigências } \\
\hline Álcalis solúveis (disponíveis) & & - & - \\
\hline Óxido de sódio $\left(\mathrm{Na}_{2} \mathrm{O}\right)$ & 0,08 & - & - \\
\hline Óxido de potássio $\left(\mathrm{K}_{2} \mathrm{O}\right)$ & 0,70 & - & - \\
\hline Equivalente alcalino ( $\left.\mathrm{Na}_{2} \mathrm{Oeq}\right) \%$ & 0,54 & - & - \\
\hline Perda ao fogo & 0,78 & $\leq 4,5$ & NBR 5733 \\
\hline Resíduo insolúvel & 0,42 & $\leq 1,0$ & NBR 5733 \\
\hline
\end{tabular}

NOTA: $\mathrm{Na}_{2} \mathrm{Oeq}=\% \mathrm{Na}_{2} \mathrm{O}+0,658 . \% . \mathrm{K}_{2} \mathrm{O}$

Na Tabela 3, pode-se observar as características físicas e química dos aditivos. As duas bases do aditivo superplastificante utilizado são comercializadas para uso na construção civil. A alcalinidade obtida da melamina foi de $5,46 \%$ e do lignosulfonato, 3,69 \%. Os dois tipos apresentaram valor de densidade muito próximos entre si, divergindo no teor de sólido (melamina com $21,6 \%$ e o lignosulfonato com $44,8 \%$ ).

Tabela 3 - Características físicas e químicas dos aditivos.

\begin{tabular}{|c|c|c|}
\hline \multicolumn{3}{|c|}{ Característica física } \\
\hline Descrição & Melamina & Lignosulfonato \\
\hline Aparência & Líquido incolor & Líquido marrom \\
\hline Densidade $\left(\mathrm{g} / \mathrm{cm}^{3}\right)$ & 1,12 & 1,20 \\
\hline Teor de sólido (\%) & 21,60 & 44,80 \\
\hline \multicolumn{3}{|c|}{ Característica química (\%) } \\
\hline Descrição & Melamina & Lignosulfonato \\
\hline Equivalente alcalino $\left(\mathrm{Na}_{2} \mathrm{Oeq}\right) * *$ & 5,46 & 3,69 \\
\hline
\end{tabular}

A amostra de agregado possui como mineralogia principal os feldspatos (plagioclásio e microclínio) e o quartzo. Os minerais subordinados, identificados na referida amostra, são: biotita, carbonato, epidoto, apatita, sericita-muscovita e opacos. Identificou-se uma alta deformação e alteração mediana na amostra. $O$ tipo de rocha é metamórfica, sendo classificada como milonito.

No resultado de expansão, observa-se na Figura 3 que as amostras com lignosulfonato apresentaram maiores expansões quando comparada com as amostras de melamina nos teores ótimo e máximo, sendo os teores mínimos dos dois aditivos, apresentando comportamento de expansão bem próximos entre si. Observa-se que existe tendência do aumento da expansão em função do aumento do teor de superplastificante, sendo o lignosulfonato com maior expressividade de expansão. Observa-se que o tipo e teor de aditivo superplastificante, interfere no comportamento da expansão da RAA.

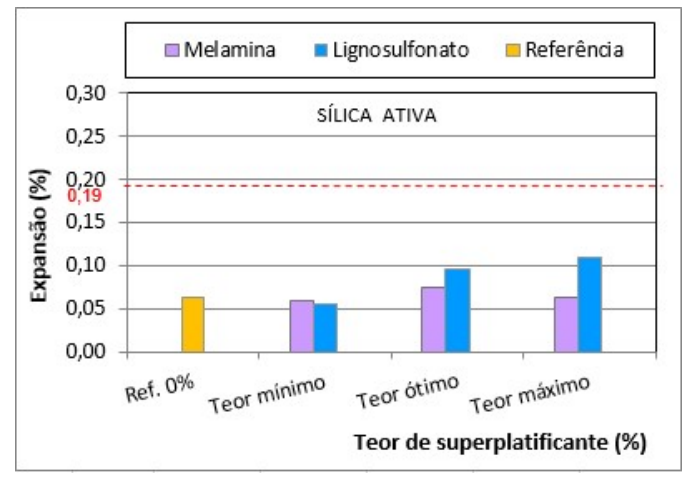

Figura 3 - Resultado de expansão aos 30 dias, com 15\% de sílica ativa e 3 teores dos aditivos superplastificantes. 
Em relação ao gel produto da RAA, sabe-se que este se deposita nos poros dos concretos e argamassas e a redução da porosidade desses aglomerados pode acarretar o aumento da expansão, devido ao fato do gel não encontrar espaço suficiente para se depositar. Esse comportamento de aumento da expansão com os maiores teores (ótimo e máximo) de superplastificantes, observado na Figura 3, pode estar ocorrendo em função da redução da porosidade dessas argamassas, pois o gel produto da RAA se deposita nos poros da argamassa e dessa forma esse gel gera tensões internas elevadas que acarretam fissuras, sendo essas fissuras responsável pelo aumento das expansões das barras. A Figura 4(a) é referente à amostra de argamassa com o teor máximo do aditivo lignosulfonato, e a Figura 4(b) se refere à amostra de argamassa com o teor máximo do aditivo melamina, em ambas foram realizadas EDS.
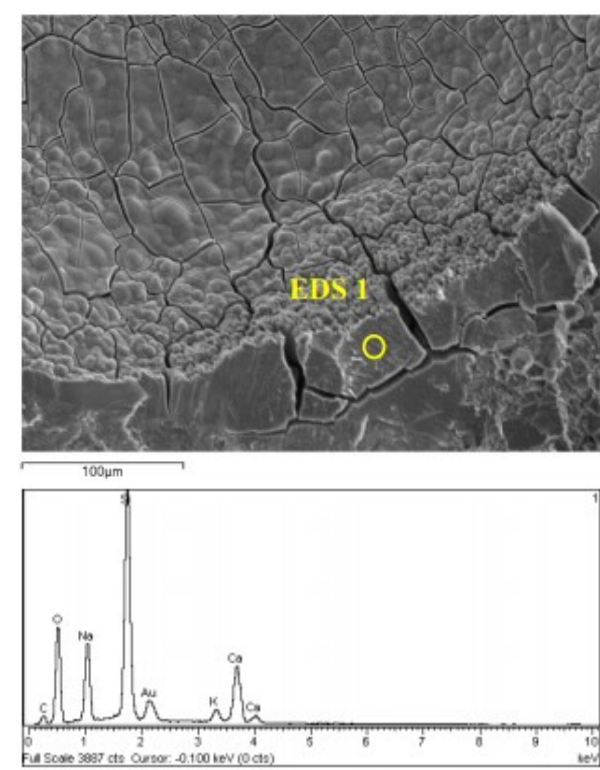

(a)
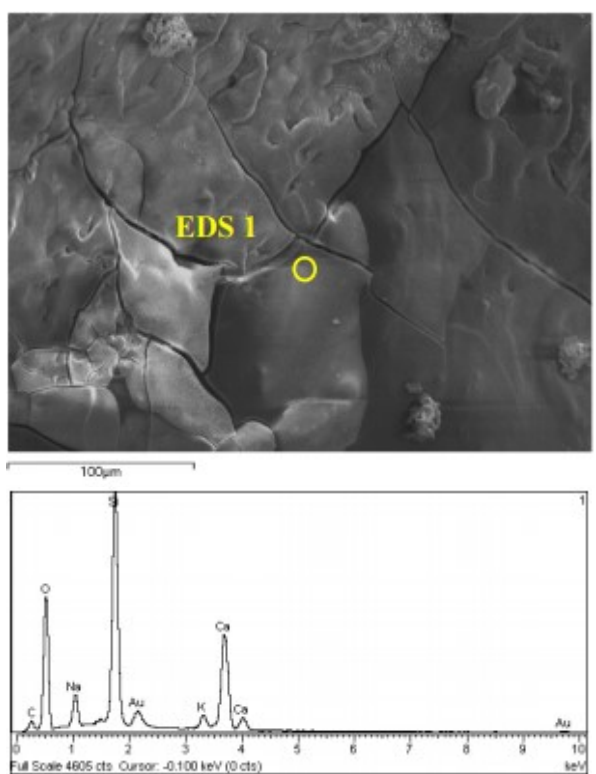

(b)

Figura 4 - (a) Amostra com lignosulfonato (teor máximo) e sílica ativa: poro preenchido com gel da RAA maciço gretado e EDS do gel; (b) Amostra com melamina (teor máximo) e sílica ativa: poro preenchido com gel gretado da RAA e EDS do gel.

Observa-se na Figura 4(a) e (b), poro com gel amorfo gretado. Os produtos encontrados nessas amostras possuem os elementos químicos silício, cálcio e potássio, mostrando-se coerente com os géis da RAA encontrados na literatura. Verificou-se a presença do gel da RAA em poros e também fora dos poros nas 2 amostras, prevalecendo o gel amorfo gretado. Em comparação com a amostra de referência, foi encontrada uma quantidade menor de poros nessas duas amostras, sendo esse possivelmente, um dos motivos para a ocorrência do aumento de tensão que gerou aumento de expansão.

Como identificou-se nas argamassas a presença de géis que provocaram expansões que originam fissuras, faz-se necessária a investigação do efeito dessas expansões nas propriedades mecânicas dessas argamassas com diferentes teores e tipos de superplastificantes, uma vez que fissuras, bem como a presença dos géis, podem contribuir para o aumento ou redução da resistência à compressão e da tração na flexão.

Na Figura 5, pode-se observar que a resistência à compressão da amostra de referência (sem aditivo superplastificante) foi elevada. Observando a resistência à compressão das amostras com os teores mínimo, ótimo e máximo da melamina, nota-se que essa resistência é diretamente proporcional aos teores de aditivos. Observando esta resistência com os 3 teores do aditivo lignosulfonato, obteve-se o mesmo comportamento da resistência à compressão do aditivo melamina, com a diferença dos valores do lignosulfonato serem menores. 


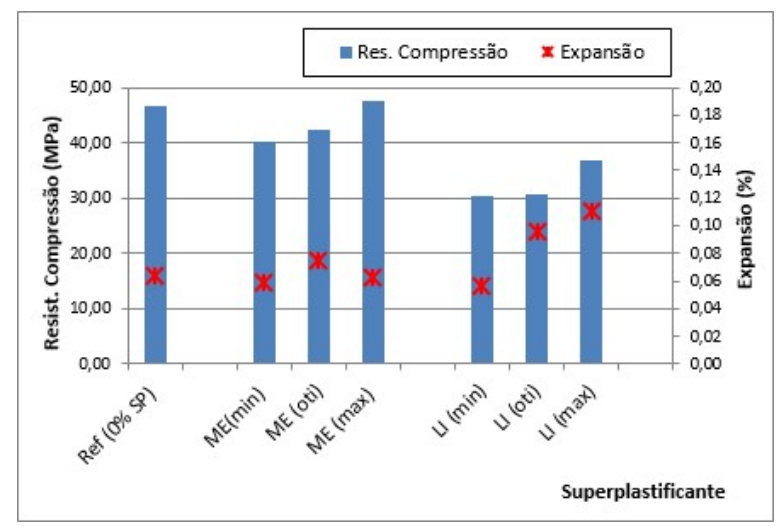

Figura 5 - Resultado da resistência à compressão versus expansão de argamassa aos 30 dias com uso de sílica ativa e os três teores dos aditivos químicos.

Quando comparada a resistência à compressão com o resultado da expansão, observa-se que a amostra com lignosulfonato apresentou comportamento diretamente proporcional, ou seja, quanto maior foi o resultado da expansão, maior foi o resultado da resistência à compressão, obtendo-se maior resistência à compressão com o teor máximo desse aditivo, e menor resistência com o teor mínimo. Comparando ainda esses dois ensaios (expansão e resistência à compressão) utilizando o superplastificante melamina, observa-se a tendência diretamente proporcional com os teores mínimo e ótimo.

Com esse resultado, percebe-se que as fissuras provenientes da expansão das barras não interferiram no comportamento observado na Figura 5 (aumento da expansão em função do aumento da resistência à compressão). Um dos fatores que, possivelmente, pode ter interferido para o aumento da resistência à compressão e para a elevação da expansão, foi a utilização dos maiores teores (ótimo e máximo) dos superplastificantes que provocaram a redução da porosidade das argamassas.

Na Figura 6, observa-se que a amostra de referência apresentou o maior valor de resistência à tração na flexão. Observando-se essa resistência com os teores mínimo, ótimo e máximo da melamina, nota-se que quanto maior o teor de superplastificante, menor é a resistência à tração na flexão, obtendo maior resistência de tração na flexão com o teor mínimo e menor resistência com o teor máximo desse aditivo. Observando essa resistência com os 3 teores de lignosulfonato, não observou-se neste aditivo o mesmo comportamento da melamina.

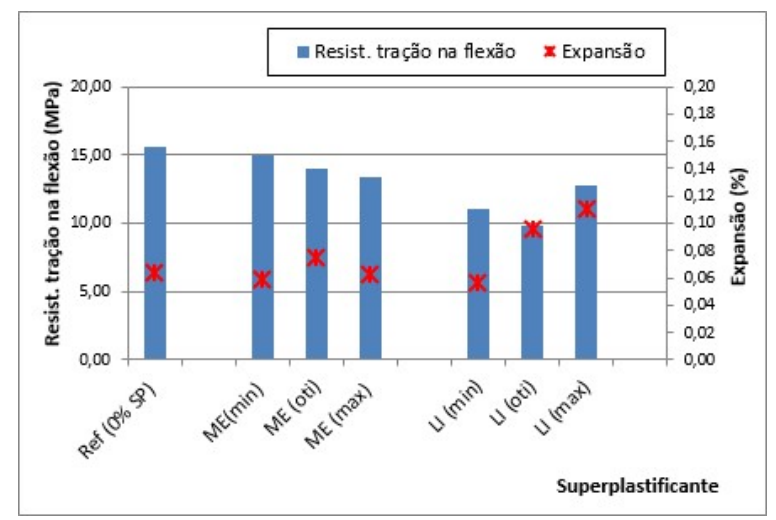

Figura 6 - Resultado da resistência à tração na flexão versus expansão de argamassa aos 30 dias com uso de sílica ativa e os três teores dos aditivos químicos.

Quando comparada a resistência à tração na flexão com o resultado da expansão, observa-se que a amostra com melamina apresentou tendência de comportamento diretamente proporcional com os teores ótimo e máximo desse aditivo. As fissuras provocadas pela expansão não tiveram influência direta na resistência à tração na flexão, pois investigou-se se as maiores expansões acarretavam redução da resistência à tração na flexão, o que não ocorreu com a maioria das amostras. 


\section{Conclusões}

Observa-se que o tipo e teor de aditivo superplastificante, interfere no comportamento da expansão da RAA. Verificou-se a presença do gel da RAA em poros e também fora destes, nas amostras de argamassa com os superplastificantes, prevalecendo o gel amorfo gretado.

Comparada com a amostra de referência, foi encontrada uma quantidade menor de poros nessas duas amostras, sendo esse possivelmente, um dos motivos para a ocorrência do aumento de tensão que gerou aumento de expansão. A redução da quantidade de poros é um dos fatores que pode ter interferido para o aumento da expansão das argamassas com os maiores teores de superplastificantes.

O resultado de expansão apresentou tendência diretamente proporcional ao resultado de resistência à compressão nas amostras dos dois superplastificantes. Um dos fatores que, possivelmente, pode ter acarretado tanto o aumento da resistência à compressão, quanto a elevação da expansão, foi a utilização dos maiores teores (ótimo e máximo) dos superplastificantes que provocaram a redução da porosidade das argamassas, dificultando o gel a se depositar nos poros e com isso elevar as expansões.

As fissuras provocadas pela expansão não tiveram influência direta na resistência à tração na flexão, pois se investigou se as maiores expansões acarretavam redução da resistência à tração na flexão, o que não ocorreu com a maioria das amostras. Diante disso, conclui-se que a resistência à tração na flexão não apresentou correlação com a expansão.

\section{Referências Bibliográficas}

ASSOCIAÇÃO BRASILEIRA DE NORMAS TÉCNICAS. NBR 11768/2011: Aditivos químicos para concreto de cimento Portland - Requisitos. ABNT/CB-18, 2011.

ASSOCIAÇÃO BRASILEIRA DE NORMAS TÉCNICAS. NBR 15577-3/2008: Análise petrográfica para verificação da potencialidade reativa de agregados em presença de álcalis do concreto. Rio de Janeiro, 2008.

ASSOCIAÇÃO BRASILEIRA DE NORMAS TÉCNICAS. NBR 15577-5/2008: Determinação da mitigação da expansão em barras de argamassa pelo método acelerado. Rio de Janeiro, 2008.

CHANG, L.; MICHAEL, D. A. T.; JASON, H. I. A mechanistic study on mitigation of alkali-silica reaction by fine lightweight aggregates. Cement and Concrete Research, 104. 2018.

FARIAS, L. A.; HASPARYK, N. P.; ANDRADE, M. A. S. Estudo preliminar de diferentes bases de aditivos e adições na RAA. In: CONGRESSO BRASILEIRO DO CONCRETO. Anais... IBRACON. 2007.

GAUTAM, B. P.; PANESAR, D. K.; SHEIKH, S. A.; VECCHIO, F. J. Multiaxial expansion-stress relationship for alkali silica reaction-affected concrete. ACI Materials Journal, 114. 2017.

HASHEMI, A.; HORST, M.; KURTIS, K. E.; DONNELL, K. M.; ZOUGHI, R. Comparison of Alkali-silica Reaction Gel Behavior in Mortar at Microwave Frequencies. IEEE Transactions on Instrumentation and Measurement, 64. 2015.

LEEMANN, A.; LOTHENBACH, B.; THALMANN, C. Influence of superplasticizers on pore solution composition and on expansion of concrete due to alkali-silica reaction. Construction and Building Materials. 25; 2011.

MULTON, S. Evaluation expérimentale et théorique des effets mécaniques de l'alcali-reaction sur des structures modèles. ERLPC (OA 46), Laboratoire Central des Ponts et Chaussées: pp 423. 2004.

NAGROCKIENĖ, D.; RUTKAUSKAS, A. The effect of fly ash additive on the resistance of concrete to alkali silica reaction. Construction and Building Materials, 201, 2019.

RAMACHANDRAN, V. S. Admixture interactions in concrete. IN: RAMACHANDRAN, V. S. Concrete Admixture Handbook: Properties Science and Technology. Canada: 2nd edition, p. 95-136. 1995.

SILVA, C. F. C.; MONTEIRO, E. C. B.; GUSMÃO, A. D. Análisis de métodos de prevención de la reacción álcaliagregado: Análisis petrográfico y método acelerado para barras de mortero. Ingeniería, Revista Académica de la FI-UADY, 15-1, p 9-17, ISSN: 1665-529-X; 2011.

TOSUN, K.; FELEKOGLU, B.; BARADAN, B. The effect of cement alkali content on ASR susceptibility of mortars incorporating admixtures. Building and Environment. 2007. 